\title{
ON LAGRANGE-HERMITE INTERPOLATION*
}

\author{
J. F. TRAUB $\dagger$
}

1. Introduction. Let the $p(n+1)$ numbers $y_{i}^{(m)}, 0 \leqq i \leqq n, 0 \leqq m$ $\leqq p-1$, be given. It is well known that there exists a unique polynomial $P_{n, p}(t)$ of degree $p(n+1)-1$ such that

$$
P_{n, p}^{(m)}\left(x_{i}\right)=y_{i}{ }^{(m)}, \quad 0 \leqq i \leqq n, \quad 0 \leqq m \leqq p-1 .
$$

A classical problem is to find a formula for $P_{n, p}(t)$ in the form

$$
P_{n, p}(t)=\sum_{i=0}^{n} \sum_{m=0}^{p-1} C_{m, i}^{n, p}(t) y_{i}{ }^{(m)} .
$$

The conditions on the $C_{m, i}^{n, p}(t)$ are that

$$
D_{t}{ }^{j} C_{m, i}^{n, p}\left(x_{r}\right)=\delta_{j, m} \delta_{r, i}, \quad 0 \leqq r \leqq n, \quad 0 \leqq j \leqq p-1,
$$

where $D_{t} \equiv d / d t$ and $\delta_{j, m}$ is a Kronecker symbol. These conditions are used by Householder [5, pp. 193-195] to derive the formulas for $p=1,2$. The formula for $p=3$ is given by Salzer [9]. The solution for $n=0$ is given by Taylor's formula.

Many authors have reported on the case where $p$ depends on $i$. General prescriptions for a solution in this more general case may be found in Fort [2, pp. 85-88], Greville [3], Hermite [4], Krylov [6, pp. 45-49], Kuntzmann [7, pp. 167-169], and Spitzbart [12]; but these prescriptions do not determine the structure of the interpolating polynomial. By restricting ourselves to the case where $p$ is independent of $i$, which is the most important case in practice, we can determine the structure. Salzer [10] discovered some of the properties of $P_{n, p}(t)$ by semiempirical means.

We shall obtain, by a partial fraction expansion, a solution of surprising simplicity. [See (3.6), (3.7), or (3.8).] The solution depends upon the Bell polynomials which we now discuss.

2. Bell polynomials. Let $g=g(t)$ and define $B_{n}$ by

$$
e^{-\omega g} D_{t}{ }^{n} e^{\omega g}=B_{n}(\omega)=B_{n}\left(\omega ; g_{1}, \cdots, g_{n}\right), \quad g_{i} \equiv g^{(i)} .
$$

$B_{n}$ is a polynomial in $\omega$ with coefficients which are polynomials in $g_{i}$. Define $U_{n, k}$ by

$$
B_{n}\left(\omega ; g_{1}, \cdots, g_{n}\right)=\sum_{k=0}^{n} U_{n, k}\left(g_{1}, \cdots, g_{n-k+1}\right) \omega^{k} .
$$

* Received by the editors May 4, 1964, and in revised form July 2, 1964.

$\dagger$ Bell Telephone Laboratories, Incorporated, Murray Hill, New Jersey. 
Then

$$
U_{n, k}=\frac{1}{k !} D_{\omega}^{k} B_{n}(0)
$$

The $B_{n}\left(1 ; g_{1}, \cdots, g_{n}\right)$ were studied by Bell [1]. (See also Schlömilch [11, p. 4].) An explicit formula for $B_{n}$ is

$$
B_{n}=n ! \sum \omega^{j} \prod_{i=1}^{n} \frac{1}{b_{i} !}\left(\frac{g_{i}}{i !}\right)^{b_{i}},
$$

where $j=\sum_{i=1}^{n} b_{i}$ and where the sum is taken over all nonnegative integers $b_{i}$ for which $\sum_{i=1}^{n} i b_{i}=n$.

Let $F(t)=f[g(t)]$. Then

$$
F^{(n)}=\sum_{k=0}^{n} f^{(k)} U_{n, k}\left(g^{\prime}, \cdots, g^{(n-k+1)}\right),
$$

or

$$
F^{(n)}=B_{n}\left(f ; g^{\prime}, \cdots, g^{(n)}\right), \quad \quad f^{(k)} \equiv f^{k} .
$$

Generating functions and symbolic recurrence relations for the Bell polynomials may be found in Bell [1] and Riordan [8, pp. 35-38, 45-48]. The first five $B_{n}$ are:

$$
\begin{aligned}
& B_{0}=1, \\
& B_{1}=\omega g_{1}, \\
& B_{2}=\omega^{2} g_{1}^{2}+\omega g_{2}, \\
& B_{3}=\omega^{3} g_{1}^{3}+3 \omega^{2} g_{1} g_{2}+\omega g_{3}, \\
& B_{4}=\omega^{4} g_{1}^{4}+6 \omega^{3} g_{1}{ }^{2} g_{2}+\omega^{2}\left(4 g_{1} g_{3}+3 g_{2}{ }^{2}\right)+\omega g_{4} .
\end{aligned}
$$

3. The formula for the interpolatory polynomial. Let $P(t) / Q(t)$ be a proper rational function and let $Q(t)$ have a zero of multiplicity $p$ at $x_{i}$. Let

$$
\begin{aligned}
& \frac{1}{Q(t)}=\sum_{j=1}^{p} \frac{\alpha_{p, j}}{\left(t-x_{i}\right)^{j}}+\eta(t), \\
& \frac{P(t)}{Q(t)}=\sum_{j=1}^{p} \frac{\beta_{p, j}}{\left(t-x_{i}\right)^{j}}+\lambda(t) .
\end{aligned}
$$

Then it is easy to show that

$$
\beta_{p, p-j}=\sum_{k=0}^{j} \alpha_{p, p-k} \frac{P^{(j-k)}\left(x_{i}\right)}{(j-k) !} .
$$


This result is the key to the solution of the Lagrange-Hermite interpolation problem. It permits us to write the interpolatory polynomial as a linear combination of the $y_{i}^{(m)}$.

Let

$$
\begin{aligned}
\pi(t) & =\prod_{i=0}^{n}\left(t-x_{i}\right), \quad Q(t)=\pi^{p}(t), \\
R_{i}(t) & =\frac{\pi(t)}{t-x_{i}}, \quad L_{i}(t)=\frac{R_{i}(t)}{R_{i}\left(x_{i}\right)} .
\end{aligned}
$$

We calculate the contribution to $P_{n, p}(t)$ due to $x_{i}$ and then sum on $i$. We have

$$
\begin{aligned}
& P_{n, p}(t) \equiv Q(t) \frac{P_{n, p}(t)}{Q(t)}=Q(t) \sum_{j=1}^{p} \frac{\beta_{p, i, j}}{\left(t-x_{i}\right)^{j}}+\rho(t), \\
& \beta_{p, i, p-j}=\sum_{k=0}^{j} \alpha_{p, i, p-k} \frac{P^{(j-k)}\left(x_{i}\right)}{(j-k) !}, \quad . \quad \alpha_{p, i, p-k}=\frac{1}{k !} D_{t}{ }^{k} R_{i}{ }^{-p}\left(x_{i}\right) .
\end{aligned}
$$

Using

$$
P^{(j-k)}\left(x_{i}\right)=y_{i}{ }^{(j-k)},
$$

we obtain, after some manipulation,

$$
\begin{aligned}
P_{n, p}(t)=L_{i}{ }^{p}(t) & \sum_{m=0}^{p-1} y_{i}{ }^{(m)} \frac{\left(t-x_{i}\right)^{m}}{m !} \\
& \cdot \sum_{\nu=0}^{p-1-m} \frac{\left(t-x_{i}\right)^{\nu}}{\nu !} R_{i}{ }^{p}\left(x_{i}\right) D_{t}{ }^{\nu} R_{i}{ }^{-p}\left(x_{i}\right)+\rho(t) .
\end{aligned}
$$

Let

$$
S_{\nu} \equiv S_{\nu}\left(x_{i}\right)=(-1)^{\nu}(\nu-1) ! \sum_{r=0 ; r \neq i}^{n} \frac{1}{\left(x_{i}-x_{r}\right)^{\nu}} .
$$

It follows from (2.1), (3.2), and (3.4) that

$$
R_{i}{ }^{p}\left(x_{i}\right) D_{t}{ }^{\nu} R_{i}{ }^{-p}\left(x_{i}\right)=B_{v}\left(p ; S_{1}, \cdots, S_{v}\right) .
$$

Using (3.5) and adding the contributions from all the $x_{i}$, we obtain as a solution to our problem

$$
\begin{aligned}
P_{n, p}(t)=\sum_{i=0}^{n} L_{i}{ }^{p}(t) \sum_{m=0}^{p-1} \frac{\left(t-x_{i}\right)^{m}}{m !} y_{i}{ }^{(m)} \sum_{\nu=0}^{p-1-m} \frac{\left(t-x_{i}\right)^{\nu}}{\nu !} & \\
& \cdot B_{\nu}\left(p ; S_{1}, \cdots, S_{\nu}\right) .
\end{aligned}
$$

Thus the essence of the $p$ th order Lagrange-Hermite formula is contained in the $B_{\nu}\left(p ; S_{1}, \cdots, S_{\nu}\right), 0 \leqq \nu \leqq p-1$. Let

$$
G_{p, i, m}=\sum_{\nu=0}^{p-1-m} \frac{\left(t-x_{i}\right)^{\nu}}{\nu !} B_{\nu}\left(p ; S_{1}, \cdots, S_{\nu}\right) .
$$


Observe that $G_{p, i, m}$ may be obtained from the polynomial $G_{p, i, 0}$ by truncating the highest $m$ terms. Hence for each $p, P_{n, p}(t)$ may be easily obtained from $G_{p} \equiv G_{p, i, 0}$. The first five $G_{p}$ are:

$$
\begin{aligned}
G_{1}=1 & \\
G_{2}=1 & +\left(t-x_{i}\right) 2 S_{1}, \\
G_{3}=1 & +\left(t-x_{i}\right) 3 S_{1}+\frac{1}{2}\left(t-x_{i}\right)^{2}\left[3^{2}{S_{1}}^{2}+3 S_{2}\right] \\
G_{4}=1 & +\left(t-x_{i}\right) 4 S_{1}+\frac{1}{2}\left(t-x_{i}\right)^{2}\left[4^{2} S_{1}{ }^{2}+4 S_{2}\right] \\
& +\frac{1}{6}\left(t-x_{i}\right)^{3}\left[4^{3} S_{1}^{3}+3 \cdot 4^{2} S_{1} S_{2}+4 S_{3}\right] \\
G_{5}=1 & +\left(t-x_{i}\right) 5 S_{1}+\frac{1}{2}\left(t-x_{i}\right)^{2}\left[5^{2} S_{1}{ }^{2}+5 S_{2}\right] \\
& +\frac{1}{6}\left(t-x_{i}\right)^{3}\left[5^{3} S_{1}^{3}+3 \cdot 5^{2} S_{1} S_{2}+5 S_{3}\right] \\
& +\frac{1}{24}\left(t-x_{i}\right)^{4}\left[5^{4} S_{1}^{4}+6 \cdot 5^{3} S_{1}{ }^{2} S_{2}+5^{2}\left(4 S_{1} S_{3}+3 S_{2}{ }^{2}\right)+5 S_{4}\right] .
\end{aligned}
$$

Equation (3.6) may be written in a number of other ways. Let

$$
T_{\nu} \equiv T_{\nu}\left(x_{i}\right)=(\nu-1) ! \sum_{r=0 ; r \neq i}^{n}\left(\frac{x_{i}-t}{x_{i}-x_{r}}\right)^{\nu} .
$$

Then

$$
P_{n, p}(t)=\sum_{i=0}^{n} L_{i}{ }^{p}(t) \sum_{m=0}^{p-1} \frac{\left(t-x_{i}\right)^{m}}{m !} y_{i}{ }^{(m)} \sum_{\nu=0}^{p-1-m} \frac{1}{\nu !} B_{\nu}\left(p ; T_{1}, \cdots, T_{\nu}\right) .
$$

Let

$$
H_{p, i, m, k}=\sum_{\nu=k}^{p-1-m} \frac{U_{\nu, k}}{\nu !}\left(T_{1}, \cdots, T_{\nu-k+1}\right) .
$$

Then

$$
P_{n, p}(t)=\sum_{i=0}^{n} L_{i}{ }^{p}(t) \sum_{m=0}^{p-1} \frac{\left(t-x_{i}\right)^{m}}{m !} y_{i}^{(m)} \sum_{k=0}^{p-1-m} H_{p, i, m, k} p^{k} .
$$

A formula for $P_{n, p}(t)$ in which the coefficients are polynomials in the $L_{i}^{(j)}\left(x_{i}\right)$ may be obtained as follows. Let

$$
R_{i}^{-p}(t)=f[g(t)], \quad f(u)=u^{-p}, \quad g(t)=R_{i}(t) .
$$

Then using (2.2), and with $L^{(j)} \equiv L_{i}^{(j)}\left(x_{i}\right)$,

$$
R^{p}\left(x_{i}\right) D_{t}{ }^{\nu} R^{-p}\left(x_{i}\right)=\sum_{k=0}^{\nu}(-1)^{k} k ! C(p+k-1, k) U_{\nu, k}\left(L^{\prime}, \cdots, L^{(\nu-k+1)}\right) .
$$

Hence

$$
\begin{aligned}
& P_{n, p}(t)= \sum_{i=0}^{n} L_{i}{ }^{p}(t) \sum_{m=0}^{p-1} \frac{\left(t-x_{i}\right)^{m}}{m !} y_{i}^{(m)} E_{p, i, m}, \\
& E_{p, i, m}= \sum_{\nu=0}^{p-1-m} \frac{\left(t-x_{i}\right)^{\nu}}{\nu !} \sum_{k=0}^{\nu}(-1)^{k} k ! C(p+k-1, k) \\
& \cdot U_{\nu, k}\left(L^{\prime}, \cdots, L^{(\nu-k+1)}\right) .
\end{aligned}
$$


Observe that $E_{p, \imath, m}$ may be obtained from the polynomial $E_{p, i, 0}$ by truncating the highest $m$ terms. Hence for each $p, P_{n, p}(t)$ may be easily obtained from $E_{p} \equiv E_{p, i, 0}$. The first five $E_{p}$ are

$$
\begin{aligned}
E_{1}=1 & \\
E_{2}=1 & +\left(t-x_{i}\right)\left[-2 L^{\prime}\right], \\
E_{3}=1 & +\left(t-x_{i}\right)\left[-3 L^{\prime}\right]+\frac{1}{2}\left(t-x_{i}\right)^{2}\left[-3 L^{\prime \prime}+12\left(L^{\prime}\right)^{2}\right], \\
E_{4}=1 & +\left(t-x_{i}\right)\left[-4 L^{\prime}\right]+\frac{1}{2}\left(t-x_{\imath}\right)^{2}\left[-4 L^{\prime \prime}+20\left(L^{\prime}\right)^{2}\right] \\
& +\frac{1}{6}\left(t-x_{i}\right)^{3}\left[-4 L^{\prime \prime \prime}+60 L^{\prime} L^{\prime \prime}-120\left(L^{\prime}\right)^{3}\right], \\
E_{5}=1 & +\left(t-x_{i}\right)\left[-5 L^{\prime}\right]+\frac{1}{2}\left(t-x_{\imath}\right)^{2}\left[-5 L^{\prime \prime}+30\left(L^{\prime}\right)^{2}\right] \\
& +\frac{1}{6}\left(t-x_{i}\right)^{3}\left[-5 L^{\prime \prime \prime}+90 L^{\prime} L^{\prime \prime}-210\left(L^{\prime}\right)^{3}\right] \\
& +\frac{1}{24}\left(t-x_{i}\right)^{4}\left[-5 L^{(4)}+120 L^{\prime} L^{\prime \prime \prime}+90\left(L^{\prime \prime}\right)^{2}-1260\left(L^{\prime}\right)^{2} L^{\prime \prime}\right. \\
& \left.+1680\left(L^{\prime}\right)^{4}\right] .
\end{aligned}
$$

As far as calculation with these formulas is concerned, observe that

$$
L_{i}^{(j)}\left(x_{i}\right)=\frac{R_{i}{ }^{(j)}\left(x_{i}\right)}{R_{i}\left(x_{i}\right)} .
$$

The $R_{i}{ }^{(j)}\left(x_{\imath}\right), j \geqq 0$, may be obtained from $\pi(t)$ by repeated synthetic division.

4. Some applications. The interpolation formula may be used to generalize the Cauchy relations,

$$
t^{j} \equiv \sum_{i=0}^{n} x_{i}{ }^{j} L_{i}(t), \quad j=0,1, \cdots, n .
$$

Corresponding to the case $j=0$, we have the following generalization.

$$
1 \equiv \sum_{i=0}^{n} L_{i}^{p}(t) \sum_{\nu=0}^{p-1} \frac{\left(t-x_{i}\right)^{\nu}}{\nu !} B_{\nu}\left(p ; S_{1}, \cdots, S_{\nu}\right) .
$$

Since the leading coefficient of $t$ on the right side of (4.1) vanishes,

$$
\sum_{i=0}^{n} \frac{1}{\left[\pi^{\prime}\left(x_{i}\right)\right]^{p}} B_{p-1}\left(p ; S_{1}, \cdots, S_{p-1}\right)=0 .
$$

This generalizes

$$
\sum_{i=0}^{n} \frac{1}{\pi^{\prime}\left(x_{i}\right)}=0 .
$$

We can derive a formula for the confluent divided difference with the same number of repetitions of all arguments, $f\left[x_{0}, p ; x_{1}, p ; \cdots ; x_{n}, p\right]$. 
(This notation is introduced in Traub [13, pp. 241-242].) Since this divided difference is the coefficient of the highest degree term in (3.6), we obtain

$$
\begin{aligned}
f\left[x_{0}, p ; x_{1}, p ; \cdots ; x_{n}, p\right]=\sum_{m=0}^{p-1} \frac{B_{p-1-m}\left(p ; S_{1}, \cdots, S_{p-1-m}\right)}{m !(p-1-m) !} \\
\cdot \sum_{i=0}^{n} \frac{f^{(m)}\left(x_{i}\right)}{\left[\pi^{\prime}\left(x_{i}\right)\right]^{p}} .
\end{aligned}
$$

\section{REFERENCES}

[1] E. T. BeLL, Exponential polynomials, Ann. of Math., 35 (1934), pp. 258-277.

[2] T. Font, Finite Differences, Clarendon Press, Oxford, 1948.

[3] T. N. E. Greville, A generalization of Waring's formula, Ann. Math. Statist., 15 (1944), pp. 218-219.

[4] C. Hermite, Sur la formule d'interpolation de Lagrange, J. Reine Angew. Math., 84 (1878), pp. 70-79.

[5] A. S. Housenolder, Principles of Numerical Analysis, McGraw-Hill, New York, 1953.

[6] V. I. KRYLov, Approximate Calculation of Integrals, Macmillan, New York, 1962.

[7] J. Kuntzmann, Méthodes Numériques Interpolation-Dérivées, Dunod, Paris, 1959.

[8] J. Riondan, An Introduction to Combinatorial Analysis, Wiley, New York, 1958.

[9] H. E. SAlzer, Formulae for hyperosculatory interpolation, direct and inverse, Quart. J. Mech. Appl. Math., 12 (1959), pp. 100-110.

[10] — Hermite's general osculatory interpolation formula, this Journal, 8 (1960), pp. 18-27.

[11] O. Schlömılch, Compendium der Höheren Analysis, II, Friedrich Vieweg und Sohn, Braunschweig, 1895.

[12] A. Spitzbart, A generalization of Hermite's interpolation formula, Amer. Math. Monthly, 67 (1960), pp. 42-46.

[13] J. F. Traub, Iterative Methods for the Solution of Equations, Prentice-Hall, Englewood Cliffs, New Jersey, 1964. 